\title{
Miranda
}

Revue pluridisciplinaire du monde anglophone /

Multidisciplinary peer-reviewed journal on the English-

speaking world

$14 \mid 2017$

Early American Surrealisms, 1920-1940 / Parable Art

\section{Notre Top 11 des films anglophones de 2016}

David Roche et Vincent Souladié

\section{OpenEdition}

\section{Journals}

Édition électronique

URL : http://journals.openedition.org/miranda/10343

DOI : 10.4000/miranda.10343

ISSN : 2108-6559

Éditeur

Université Toulouse - Jean Jaurès

Référence électronique

David Roche et Vincent Souladié, « Notre Top 11 des films anglophones de 2016 », Miranda [En ligne],

14 | 2017, mis en ligne le 19 avril 2017, consulté le 16 février 2021. URL : http://

journals.openedition.org/miranda/10343; DOI : https://doi.org/10.4000/miranda.10343

Ce document a été généré automatiquement le 16 février 2021.

\section{$\Theta \Theta \Theta \Theta$}

Miranda is licensed under a Creative Commons Attribution-NonCommercial-NoDerivatives 4.0 International License. 


\title{
Notre Top 11 des films anglophones de 2016
}

\author{
David Roche et Vincent Souladié
}

2016 aura été une très grande année pour le cinéma anglophone, et ce dans toutes ses ramifications. Certains blockbusters n'ont pas été au rendez-vous - les tant attendus Suicide Squad (USA, David Ayer) et Batman v. Superman: Dawn of Justice (USA, Zack Snyder) -, d'autres ont surpris - Captain America : Civil War (USA, Anthony et Joe Russo) et son discours métafictionnel sur la dimension éthique du récit de superhéros, et surtout le provocateur et ultra-méta Deadpool (USA, Tim Miller). Des cinéastes confirmés ont déçu - les frères Coen avec Hail, Caesar!-, d'autres ne nous ont pas convaincu tout en accumulant les récompenses - La La Land (USA / Hong Kong, Damien Chazelle) dont les détracteurs sont allés jusqu'à dire que le talentueux cinéaste n'avait pas compris grand-chose au genre de la comédie musicale. Avec Certain Women (USA), Kelly Reichardt livre une adaptation subtile des nouvelles de Maile Meloy, son intérêt pour le quotidien de femmes ordinaires s'inscrivant dans la filiation de la regrettée Chantal Akerman. Le renouveau du film d'horreur américain, annoncé par l'envoutant It Follows (David Robert Mitchell, 2014), semble se confirmer avec The Witch (USA/UK/ Canada/Brazil, Robbert Eggers), remarqué à Sundance, et Green Room (USA, Jeremy Saulnier), deux films qui combinent propos politiques - critique des origines puritaines des États-Unis dans la lignée des fictions de Nathaniel Hawthorne pour le premier, critique des mouvements White Supremacist actuels pour l'autre - et recherche plastique - gothique pictural pour le film d'Eggers, et travail sur la couleur justifié par le lieu principal dans le film de Saulnier. Après le très drôle What We Do in the Shadows (Nouvelle Zélande / USA, 2014), le cinéaste et acteur néo-zélandais Taika Waititi, qui est aussi un adepte du film de genre, livre avec Hunt for the Wilderpeople (Nouvelle Zélande) un récit de voyage et de filiation rafraichissant en forme de conte, sous-tendu lui aussi par une réflexion sur l'histoire ethnique de la Nouvelle Zélande. Avec Captain Fantastic (USA), le jeune cinéaste américain Matt Ross livre lui aussi un film rafraichissant, avec un rôle taillé sur mesure pour Viggo Mortensen et une fin qui parvient à déjouer les écueils idéologiques dans lesquels navigue le film. Whit Stillman transforme une longue nouvelle de Jane Austen, “Lady Susan” renommée Love \& Friendship (Irlande/France/ 
Pays-Bas), en une satire incisive proche du Vanity Fair de W.P. Thackeray, et Sing Street (Irlande/UK/USA, John Carney) réalise la prouesse de raconter l'histoire touchante d'un groupe de rock dont chaque titre est une quasi-reprise du succès du moment sans condescendance aucune envers ces jeunes personnages. Quant au cinéaste britannique David Mackenzie, ses nominations aux Oscars témoignent de la réussite de son passage outre-Atlantique, avec Hell or High Water (USA), heist movie proposant lui aussi une critique du contexte social, économique et historique de l'état du Texas dans lequel il se déroule. Le cinéma d'animation grand public a aussi connu de beaux succès, avec les productions Disney récompensées aux Oscars - Moana (USA, Ron Clements, Don Hall, John Musker et Chris Williams) et Zootopia (USA, Byron Howard, Rich Moore et Jared Bush) -, mais aussi le grand oublié, Kubo and the Two Strings (USA, Travis Knight).

2 Les onze films qui suivent ne sont pas tous des films parfaits, mais tous nous ont séduit par leurs ambitions et leurs réussites respectives. Ils nous ont aussi séduit parce qu'en eux circule un souffle d'utopie, bienvenu après les récits apocalyptiques post-11 septembre. Tous ces films cherchent à déceler le «bon » dans l'humain, quand bien même reconnaissent-ils la noirceur du monde contemporain.

\section{American Honey (UK/USA, Andrea Arnold)}

3 Avec son quatrième long métrage, la cinéaste britannique a du se dire que, quitte à faire un film américain, autant aller jusqu'au bout des choses en réalisant un road movie qui se déroule dans le midwest, et dont le titre est tiré d'une chanson country, écrite et interprétée par le groupe Lady Antebellum (2010). Le film se veut une exploration de la jeunesse américaine contemporaine et de son environnement à travers une exploration des codes du cinéma américain. Le récit multiplie ainsi les fausses pistes (s'agit-il d'un réseau de pornographie ou de prostitution? les trahisons vont-elles conduire à une fin explosive ?) qu'il déjoue sans fanfare. Comme Lynne Ramsay, Arnold est à la fois une naturaliste et une esthète, la réalité étant toujours médiatisée par la subjectivité de ses héroïnes. Le contexte américain fait d'ailleurs ressortir l'influence d'un Gus van Sant et surtout d'un Larry Clark. American Honey s'affirme comme une adaptation avec variation du magnifique Fish Tank (UK/Pays-Bas, 2009): comme Mia, Star est un personnage fort dont la vision et le corps sont néanmoins conditionnés par sa classe sociale, mais contrairement à Mia, elle trouve rapidement sa place au sein d'un groupe de jeunes, menés par la mystérieuse Krystal et son lieutenant Jake. Et si l'équipe de Krystal connait, certes, quelques tensions, elle constitue néanmoins une famille de substitution pour ces jeunes marginaux, qui communient physiquement et musicalement (la bande son est d'ailleurs magnifique), les scènes de chant et de danse étant sans doute les moments les plus jubilatoires du film. Et c'est aussi là où American Honey se démarque du cinéma américain, cette fois-ci indépendant: parce que si l'absence de condescendance vis-à-vis des personnages rappelle le ton des films de Clark, Arnold met en avant l'utopie américaine qui gravite autour du personnage de Star. Non seulement ces jeunes n'ont-ils rien de menaçant, mais ils représentent un groupe hétérogène, aux goûts musicaux éclectiques et au parler influencé par la culture hiphop, et cela malgré le fait qu'ils soient tous blancs (ou presque car l'identité raciale de Star n'est jamais en question). Le point culminant de cette utopie, c'est la rencontre des wannabes avec des personnages afro-américains qui n'aboutit à rien d'autre qu'à une conversation sympathique. Si les premiers films américains de nombreux cinéastes 
européens contemporains sont souvent des attaques en règle contre les Etats-Unis Bread \& Roses (UK/France/Allemand/Espagne/Italie/Suisse, Ken Loach, 2000), Dancer in the Dark (Danmark/Espagne/Allemagne/Pays-Bas/Italie/USA/UK/France/Suède/ Finlande/Isande/Norvège, Lars Von Trier, 2000) -, American Honey crée la surprise en évitant la vision baudrillardienne et en chantant les louanges de l'utopie et de la liberté (le motif de l'oiseau) américaines, incarnées par Star, interprétée par l'étincelante Sasha Bianca Lane, native du Texas dont les parents sont justement afro-américain (le père) et néo-zélandais (la mère). Avec American Honey, récompensé par le Prix du Jury à Cannes et vainqueur du BIFA (British Independent Film Award), Arnold, l'une des cinéastes de sa génération les plus talentueuses, consolide une œuvre déjà impressionnante.

\section{Arrival (USA, Denis Villeneuve)}

4 Réalisateur stakhanoviste remarqué, le cinéaste canadien tourne rapidement et efficacement en passant régulièrement d'un genre à l'autre. Cette démarche de cinéaste de série B est contredite par l'ambition esthétique dont ses films font preuve et par la gravité de leur ton. Villeneuve est présent dans notre top pour la deuxième année consécutive. En 2015, Sicario nous avait marqué par sa noirceur politique, couronnant un regard dur et pessimiste entamée dès ses premiers films, notamment Polytechnique (Canada, 2009). Cette année Arrival parvient à s'ouvrir à l'optimisme tout en refusant la légèreté: l'humanité est sauvée par l'universalité du langage mais au prix d'un inéluctable sacrifice. Il était au départ très risqué de revisiter le sujet maintes fois rebattu de la première rencontre sur Terre avec la vie extraterrestre et de ses conséquences. En adaptant la nouvelle "Story of Your Life» (Ted Chiang, 1998), Villeneuve et son équipe parviennent pourtant à faire preuve d'originalité en faisant le pari de la science-fiction dure et en ramenant les questionnements universels de la science-fiction sur le terrain de l'intime. L'intrigue suit le point de vue d'une universitaire linguiste chargée de décrypter le langage abscons des aliens et amenée à faire le plus difficile des choix moraux alors qu'elle est au cœur d'un complexe nouage temporel. Face aux conflits géopolitiques que dramatisent habituellement le genre et qui servent de cadre au récit, le film célèbre au contraire la collectivité des chercheurs et insiste que les solutions s'inscrivent dans la durée et non dans les ultimatum. En cela, Arrival rejoint la démarche récente de Interstellar (USA/UK/Canada/Islande, Christopher Nolan, 2014), d'ailleurs présent dans notre top 2014. Mais le film de Denis Villeneuve s'avère plus réussi encore, principalement en raison de sa douceur et de sa modestie, transmises par le style de la mise en scène, de ses qualités plastiques et de sa bande son signée de Jóhann Jóhannsson, récompensé aux Oscars: la lenteur des mouvements de caméra, la pâleur monochrome de la photographie, le travail prononcé sur le flou, autant d'effets d'implication émotionnelle réussis. On repère par ailleurs un point commun entre Interstellar et Arrival quant à leur approche formelle, c'est la double influence de Terrence Malick et de Stanley Kubrick. On repérait l'influence des Moissons du paradis / Days of Heaven (USA, Malick, 1978) chez Nolan dans la manière qu'il avait de cadrer la solennité magistrale de la pastorale américaine, ses fermes et ses champs de maïs communicant avec le cosmos. C'est le Malick lui-même imprégné de l'identité américaine institué par le cinéma classique (Vidor, Kazan, Stevens) que convoquait Interstellar. Villeneuve est moins sensible à cette représentation de l'Amérique archétypale, mais retient en revanche de Malick son atmosphère et son 
style impressionniste, la manière qu'a la caméra d'épingler les fragments fugaces et fragiles du présent, leur immanence poétique. Le visage cristallin d'Amy Adams, tout aussi remarquable ici que dans Nocturnal Animals, s'en fait l'emblème. L'influence de Kubrick se manifestait dans Interstellar à travers le modèle évident de 2001, A Space Odyssey (1968) et son ampleur métaphysique. Arrival ne passe pas lui non plus à côté de cet héritage, et retient plutôt la mise en scène du silence anxiogène et de la solitude face au mystère sidéral. On attendra désormais de l'intention formaliste manifeste de Villeneuve qu'elle installe plus franchement sa singularité.

\section{Everybody Wants Some !! (USA, Richard Linklater)}

Boyhood (USA, Richard Linklater, 2014) nous avait séduit il y a deux ans pour sa modestie et sa douceur, une forme de politesse par rapport à son sujet, au regard de l'audace inédite du projet. La fiction intimiste au long cours réussissait à se tenir dans les ornières du mélodrame et du pensum, évitant ainsi de totalement confondre la singularité du destin privé et la fresque existentielle. Les situations mises en scène par le film avançaient alors en équilibre sur une ligne ténue, entre insignifiance et universalité. Cet art de l'équilibre dramatique, Richard Linklater le démontre à nouveau dans Everybody Wants Some, dans un registre sensiblement différent. Dans Boyhood, la consistance fictionnelle du jeune personnage principal n'était jamais acquise, mais le travail temporel du film nous rendait sa présence familière et donc attachante. On a souvent présenté Everybody Wants Some comme une suite tardive de Dazed and Confused (USA, Richard Linklater, 1993); pourtant, les adolescents de ce dernier était mus par des quêtes identitaires d'un autre ordre. Au sein de la bande d'étudiants fraîchement installée sur un campus texan, dans Everybody Wants Some!!, tous affichent dès le départ leur vacuité et se comportent en caricature, à tel point que le spectateur peut se trouver facilement désarçonné par l'indécision assumée entre parodie de teen movie des années 80 , dont le film reprend les principaux topoï et la chronique au premier degré. L'idiotie des personnages et les enjeux narratifs dérisoires qui les animent ne sont pas le facteur le plus original du film, en phase avec le genre précité. En revanche, c'est en s'efforçant de ne pas favoriser l'identification classique des spectateurs aux étudiants gouailleurs que le film constitue un défi qui l'installe là encore sur le fil du rasoir. Les figures habituelles du genre sont notamment désamorcées par la béance narrative : la scène de l'entraînement au baseball ne voit aucun personnage se distinguer des autres, aucune hiérarchisation des actions, aucune amorce de drame à résoudre. Dès lors, au plaisir de redécouvrir les archétypes du teen movie américain, s'ajoute une consternation parfois malaisante devant certaines de leurs actions. La dynamique de groupe de ce film choral est en fin de compte son seul vrai sujet affirmé, et son inscription dans le temps lui donne une portée métaphysique qui rapproche ainsi le film de Boyhood. En sous-texte de cette empathie à double tranchant peut se lire une réflexion plus subtile sur le racisme insidieux des teen movies 80s. En effet, on ne voyait aucun jeune afro-américain dans la série des Porky's (Canada/ USA, Bob Clark, 1982), ni même dans les films de John Hugues, tandis que chez Linklater, en réponse, les étudiants blancs rappent eux-mêmes sur Rapper's Delight ! 


\section{Moi, Daniel Blake / I, Daniel Blake (UK/France/Belgium, Ken Loach)}

6 En gagnant la Palme d'Or en 2016, Ken Loach a rejoint le cercle fermé des doubles vainqueurs du festival (la première était pour Le vent se lève / The Wind that Shakes the Barley en 2006). Et pourtant ce dernier film de Loach ne frappe pas d'emblée comme le plus grand film du prolifique cinéaste britannique, ni même peut-être l'un de ses meilleurs. Pour qui connaît bien l'œuvre du cinéaste britannique, on y retrouve tous les ingrédients habituels: un scénario signé Paul Laverty (scénariste de Loach depuis 1996), une mise en scène sobre qui laisse respirer un jeu d'acteur naturaliste servi par des acteurs professionnels peu connus (le comique et acteur de télévision Dave Johns, l'auteure de pièces de théâtre et actrice de télévision Hayley Squires, tous deux excellents), une photographie qui installe d'emblée une ambiance terne. On peut même reprocher au film une fin un brin mélodramatique, ainsi qu'une représentation utopique des classes défavorisées (les enfants de Katie sont bien là pour démontrer l'absence de racisme et de xénophobie dans cette classe), et en conclure que le film ne constitue rien d'autre que de la propagande de gauche. Ce récit de personnages courageux pris dans l'étau d'une administration à la logique kafkaesque et pourtant ancrée dans une réalité socio-historique contient des scènes d'une intensité émotionnelle incontestable dans lequel de simples objets (une conserve de Heinz Baked Beans, une boîte à lettres) prennent en charge le désarroi de l'injustice par métonymie. Et pourtant il nous a semblé que c'était la première fois qu'un film de Loach offrait une réflexion métafictionnelle sur ce que cela signifie de produire un cinéma politique à l'heure où le libéralisme capitaliste a réussi à s'imposer non pas comme une idéologie, mais comme un état de fait. Parce que l'acte politique et esthétique (car il s'agit d'un graffiti) qui donne son titre au film se révèle comme étant dérisoire : dérisoire parce que la révolte et la souffrance sont tournées en spectacle que l'on peut immédiatement photographier et poster sur les réseaux sociaux où la force vitale sera diluée parmi les autres informations; et parce que le public qui reçoit le discours final de Daniel est composé de personnes déjà convaincues de sa validité. Bref, exactement comme le public qui se rend au cinéma pour voir les films de Loach. C'est en ce sens que I, Daniel Blake est un film politique au sens où l'entend Jacques Rancière : c'est-à-dire un film qui s'interroge sur son statut de film politique. Et à travers cet acte qu'est la production d'un film, et malgré cette conscience de ses propres limites, Loach et son équipe persistent, comme la sénatrice américaine Elizabeth Warren. Et ça même si ça fait mal, nous en avons encore besoin.

\section{Jackie (Chile/France/USA/Hong Kong, Pablo Larraín)}

7 Ce portrait en mosaïque de Jackie Kennedy recèle une complexité à laquelle le genre en vogue du biopic nous a peu habitué, genre que le cinéaste a contribué à régénérer en signant coup sur coup Jackie et le tout autant réussi Neruda (Chile/Argentine/France/ Espagne/USA, 2016). Cette complexité est d'abord d'ordre narratif et peut surprendre de la part du scénariste Noah Oppenheim, jusque-là crédité sur des franchises adolescentes comme The Maze Runner (USA/UK, Wes Ball, 2010). L'intelligence du récit vient d'abord du resserrement de son sujet biographique (depuis l'assassinat du président Kennedy jusqu'à ses funérailles), de sa résistance au didactisme (le portrait 
impressionniste l'emporte sur la leçon d'histoire), et de sa désobéissance aux lois du sensationnalisme (tout voyeurisme est évité). La narration en flashbacks depuis le point de vue de l'héroïne interviewée pour le magazine Life offre une accroche immersive qui permet de percevoir depuis l'intérieur la collusion et la confusion entre la douleur intime et l'intensité de l'événement historique. Jackie n'est en aucun cas une hagiographie, plutôt la description ambiguë d'une personnalité ambiguë saisie sur le vif par une tragédie intime instantanément confisquée par les regards médiatiques, tout autant que par la marche en avant de l'histoire, contraignant chacun de ses gestes et chacun de ses mots à être lestés d'un poids symbolique impitoyable. Le deuxième gage de complexité du film vient alors du travail magistral de Nathalie Portman, toujours en quête de superposer dans sa composition des niveaux de sens contradictoires (modulation de la voix selon les contextes, micro inflexions des traits du visage, démarche tour à tour spectrale ou assurée), de façon à construire un personnage sans cesse opaque et fuyant. La perception de chaque scène est ainsi tendue entre l'image de la fragilité et la sensation du contrôle, sans que le spectateur puisse jamais déterminer si la Jackie Kennedy du film feint la maitrise de soi pour masquer son effondrement intérieur ou si elle cherche délibérément à humaniser sa persona en mettant en scène sa vulnérabilité face au journaliste. Enfin, la complexité du film est portée par les choix formels, lesquels enrichissent la narration non chronologique d'un entrecroisement expressif d'identités stylistiques. Dans un tout autre registre que Arrival, Pablo Larraín se repait à son tour du style de Terrence Malick, dont le style confirme son influence : la caméra portée serre le visage de Jackie Kennedy dans les trouées de lumière mélancoliques du soleil couchant, le temps de ses errances silencieuses et solitaires sur les jardins de sa propriété. Cette caméra "malickienne» devient là encore "kubrickienne" lorsque Jackie est suivie en steadycam dans de longs travellings anxiogènes à la Shining (UK/USA, 1980) dans les couloirs vides de la Maison Blanche. La composition picturale du cadre, impressionniste dans les scènes en extérieur ou rigoureusement géométrique dans les décors solennels de Washington, suggère toujours l'impossibilité pour le corps de trouver désormais résidence dans les lieux qui lui étaient hier familiers. Cette question de l'expropriation du personnage par l'hostilité de son environnement est au cœur même du film, et sert de contrepoint à une reconquête identitaire par l'image. La photographie de Stéphane Fontaine (Under the Skin, Jonathan Glazer, 2013) et le format de pellicule utilisé, comparable au travail de Todd Haynes et de son chef opérateur Edward Lachman sur Carol (2015), de même que la reconstitution minutieuse des documentaires d'archives 16 millimètres, exposent la figure de Jackie à un espace de mise en scène permanent dans lequel se définit son identité. Ce statut de l'image permet à Jackie d'interroger la construction de l'histoire à travers le statut des images. Pablo Larraín reprend alors en quelque sorte les ambitions introduites par Michael Mann dans le mésestimé Ali (USA, 2001), à savoir chercher à reconstituer la trajectoire d'un personnage médiatique lancé dans la quête de sa propre identité, c'est-à-dire en fin de compte dans la quête dérisoire de son image la plus juste.

\section{Manchester by the Sea (USA, Kenneth Lonergan)}

8 Avec ce troisième long-métrage après cinq ans d'inactivité, Kenneth Lonnergan ose la noirceur du mélodrame familial, sans jamais verser dans les excès du pathos mais en l'abordant par la douceur formelle du classicisme. Lonnergan affiche la confiance 
tranquille du cinéaste qui croit en la puissance émotionnelle de son histoire et expose ses enjeux avec transparence. La construction en flashbacks progressifs ne cherche pas à prendre le spectateur à témoin de l'orfèvrerie du montage : à l'expressivité de la fresque il préfère la littéralité de la chronique, de même qu'à la subtilité d'une construction poétique, il préfère l'observation attentive des comédiens et de leur jeu. Lonnergan ne craint pas la durée et laisse respirer chaque scène avant de couper à une autre. Il n'est alors pas étonnant que le jeu taciturne de Casey Affleck ait été récompensé d'un oscar, qu'aurait sans doute mérité également Michelle Williams pour un rôle plus réservé mais inoubliable, tout comme Lucas Hedges. Cette mise en retrait du réalisateur face au drame qu'il met en scène n'est pas un signe de fadeur de la part de Lonergan, mais témoigne au contraire d'une écriture à l'économie, respectueuse de son sujet et de son spectateur. En questionnant le deuil et la culpabilité, en condensant l'étendue de la complexité des rapports familiaux (paternité contrariée puis reconquise, fraternité conflictuelle puis endeuillée, renoncement amoureux), en tissant une histoire en forme de toile d'araignée temporelle, avec ses flashbacks, ses ellipses et ses révélations savamment retenues, Manchester By The Sea respecte certes avec rigueur le cahier des charges du cinéma dramatique indépendant estampillé Sundance. On pourrait d'ailleurs y reconnaître les décors balnéaires hivernaux et mélancoliques d'Ordinary People (USA, Robert Redford, 1980), jadis oscarisé. Or, la mise en scène de Kenneth Lonergan ne se prévaut pas d'autres prétentions que celle du travail bien fait, l'efficacité, la finesse et la rigueur de la retenue contre les artifices du lyrisme.

\section{Moonlight (USA, Barry Jenkins)}

9 Moonlight a mené la reconquête des Oscars par les minorités, outrageusement laissées pour compte en 2016, jusqu'à être primé meilleur film de l'année. Cette victoire résonne forcément dans un contexte politique oppressant tout en étant bel et bien méritée. Adapté d'un récit autobiographique de Tarell Alvin McCraney, ce deuxième long métrage de Barry Jenkins est à la fois ambitieux, passionné et poétique. Il reprend les figures habituelles (le gangsta, la mère qui élève son enfant seule, le pariah) des ghetto movies du début des années 1990 - Boyz $n$ the Hood (John Singleton, 1991), Menace II Society (Albert et Allen Hughes, 1993) - mais, à l'image du magnifique Pariah (Dee Rees, 2011), leur offre d'autres issues que l'échec ou la mort, démontrant ainsi qu'il s'agit là de conventions génériques trop souvent assimilées à du réalisme. La musique orchestrale du film, dont la sensualité n'est pas sans rappeler celle d'un Michael Nyman, joue là aussi délibérément contre les clichés du genre. La structure narrative en trois parties est certes démonstrative (on pense au tic buccal que Terrone adulte reprend à Juan son mentor) mais, comme Boyhood (Richard Linklater, 2014) qui lui a sans doute servi de modèle, elle tire sa force de son emploi intelligent de l'ellipse (à commencer par la mort de Juan). Moonlight s'appuie sur un ensemble d'acteurs qui sont tous à la hauteur: Mahershala Ali, qui confirme la finesse de son jeu dans House of Cards (Netflix, 2013-) tout en élargissant sa palette, la troublante actrice britannique Naomie Harris, connue pour 28 Jours plus Tard (Danny Boyle, 2002), le jeune Ashton Sanders, révélé dans Straight Outta Compton (F. Gary Gray, 2015) et l'élégant André Holland dont le charisme crevait déjà l'écran dans The Knick (Cinemax, 2014-2016). Servie d'une photo superbe, son esthétique alliant naturalisme et effets subjectifs - regards caméra, emploi surréaliste des couleurs (le magnifique plan de la mère dans le cadre du couloir) - n'est pas sans rappeler les films de Gus van Sant ou le premier long métrage de Lynne 
Ramsay, Ratcatcher (1999). Car Moonlight marque aussi la revanche du cinéma indépendant sur l'académisme, ce que l'erreur de remise prix commise en fin de cérémonie ne fait que confirmer : qui eut cru qu'un film, produit avec un budget de \$ 5 millions et dans lequel une fin heureuse est accordée à des homosexuels des quartiers défavorisés afro-américains pourrait ravir l'Oscar à La La Land?

\section{The Neon Demon (France/Danemark/USA, Nicolas Winding Refn)}

10 La fascination procurée par The Neon Demon tient à la franche affirmation stylistique que Refn délivre une fois encore, c'est-à-dire ici au penchant littéralement vampirique de l'image. Depuis qu'il est arrivé à Hollywood, l'œuvre de Refn gagne sans effort sa cohérence artistique de l'insistance ostensible de ses obsessions visuelles (cadres contemplatifs, hyperbole chromatique et lumineuse, retenue rythmique du montage), plus vraisemblablement employées à la séduction du regard qu'à l'expression d'un discours, comme si « faire auteur » était pour Refn l'enjeu à atteindre le plus urgent et le plus immédiat. Qu'offre alors Refn en gage contre cette apparente vanité formaliste? Le parti pris esthétique est mis au service d'une réflexion sur l'articulation entre image, corps et identité : masculinité dans ses films depuis Bronson (UK, 2008) et Valhalla Rising (Danemark/UK, 2009), féminité dans The Neon Demon. Si ces influences avérées (Kenneth Anger, Stanley Kubrick) demeurent perceptibles, on pourrait reconnaître dans la représentation d'Hollywood l'influence du David Lynch de Mulholland Dr. (France/USA, 2001), et des échappées oniriques d'Alejandro Jodorowski, qu'il considère en quelque sorte comme son mentor, mais elles se trouvent toujours contrebalancées par l'ancrage générique du récit. Même la pure sensualité fétichiste de l'image, telle que magnifiée dans les années 80 par les frères Ridley et Tony Scott, et les autres réalisateurs de l'école de Londres (Alan Parker, Adrian Lyne), ne fournit pas à Refn un modèle à suivre pleinement satisfaisant, quand bien même s'inscrit-il à sa façon dans leur prolongement. Malgré leur signature stylistique très différente, c'est peut-être au cinéma de Brian de Palma que la démarche esthétique de Refn est la plus comparable, dans le sens où ils s'affirment tous deux comme des maniéristes immodérés, cherchent à imposer un équilibre entre format commercial et expérience arty, et revendiquent le mauvais goût dans lequel cet exercice les fait parfois verser. Si cette radicalité nous séduit chez Refn, c'est justement en raison de son refus du réel et de cette insistance du formalisme à vouloir épuiser à tout prix l'image dans toutes ses potentialités plastiques (ralenti, surexposition, très gros plan) pour en tirer comme un suc émotionnel pur et autonome. Dans The Neon Demon, Refn ne reprend les ingrédients horrifiques du giallo qu'en apparence pour se livrer au fond à une expérience plastique sur la physionomie froide et diaphane d'Elle Fanning. La longue séquence du shooting photo se présente en quelque sorte comme un manifeste, dans laquelle l'actrice devient l'égérie d'une conception plastique et formaliste du cinéma, corps désincarné livré aux modulations du cadre et de la lumière jusqu'à la grâce mais aussi jusqu'à l'ambiguïté de son exploitation scopique. Le milieu de la mode dans lequel se déroule l'intrigue joue comme argument en faveur d'un discours critique du cinéaste à l'égard de la vampirisation aliénante de l'image, de même que la féminité y est interrogée comme facteur de construction sociale, mais c'est encore ce troublant équilibre esthétique entre le sublime et le malaisant, déjà travaillé sur la figure masculine de Ryan Gosling 
dans Drive (USA, 2012) et Only God Forgives (Danemark/France/USA/Suède, 2014), qui impose Refn comme l'un des cinéastes hollywoodiens les plus en phase avec son temps.

\section{Nocturnal Animals (USA, Tom Ford)}

11 Ce deuxième long métrage du natif d'Austin confirme le potentiel que laissait déjà présager A Single Man (USA, 2009) - on y retrouve le thème de la mélancolie, une structure en flashback, des compositions soignées avec un goût pour le surcadrage -, mais dans un projet autrement plus abouti. Le tour de force de cette adaptation du roman d'Austin Wright (1993) est de raconter une histoire de couples, de vie ratée, d'ennui, de déception et de petite vengeance, à travers un personnage statique et quasimuet (Susan, finement interprété par Amy Adams) qui ne fait rien d'autre que lire un manuscrit. Son histoire enchâsse un récit néo-noir/détour à la fois horrible et somme toute assez banal, situé comme il se doit au Texas. Cette intrigue dégage une intensité mélodramatique en se donnant à voir comme une version idéalisée de l'intrigue par la lectrice, laquelle commet l'erreur basique de confondre l'auteur, son ancien amant (Edward Sheffield), et le personnage principal (Tony Hastings). À travers les yeux de Susan, le spectateur est alors happé par ce récit plus prenant, plus coloré, plus vibrant. Et pourtant les mises en relation entre la fiction et la vie privée ne sont que des effets de lecture pour Susan, donnant lieu pour le spectateur à des effets de compositions symétriques et de montage en parallèle qui construisent les couples Susan-Tony et Susan-Edward, tout mettant en relief la confusion opérée par la lectrice. Par ailleurs, étant donné que l'intensité dramatique du récit enchâssé prend le dessus sur le récit principal, le personnage secondaire du shériff Bobby Andes vole la vedette au rôle principal au sein même du récit secondaire, grâce à un Michael Shannon toujours aussi envoûtant. Nocturnal Animals, comme son titre l'indique, est un film d'une noirceur sans fond, mais les animaux du titre ne sont pas seulement les criminels : ce sont aussi les protagonistes caractérisés par des ambitions basses et une passivité digne des grands romans naturalistes (d'où le fait que la fin ait pu en décevoir certains). Dès lors, c'est paradoxalement le personnage mourant qui apporte une énergie vitale au film, un peu à l'image de George qui, dans A Single Man, parvenait à retrouver goût à la vie au bord du précipice.

\section{Paterson (USA/Allemagne/France, Jim Jarmusch)}

Avec son douzième long métrage, le cinéaste new-yorkais traverse la Hudson River pour explorer la ville du poète américain William Carlos Williams. L'ambition de Jarmusch est de produire une poésie filmique minimaliste, à l'image des textes de Williams mais aussi de ces successeurs, dont le plus célèbre des auteurs minimalistes Raymond Carver (le poème de la boîte d'allumettes rappelle le souci du détail que Carver hérite de Williams). Le cinéaste poursuit l'esthétique de la répétition et du détail qui le caractérise, l'influence de Yasujirô Ozu étant plus évidente que jamais dans la poétique de l'espace et du temps dévelopée. Cette filiation double - poésie américaine, cinéma japonais - est thématisée par la rencontre finale entre deux poètes, le protagoniste et son alter ego nippon; le double est d'ailleurs une figure centrale du film, avec ce running gag des jumeaux que le poète ne cesse de croiser. Mais Paterson n'est pas qu'un exercice de style : le film a un discours, sur la création, sur le couple, sur 
les États-Unis d'aujourd'hui. Paterson, le film comme le personnage éponyme, joue la fonction de témoin: de la ville, de son histoire, de ses personnalités, de ses communautés. Habitant une banlieue désertée par les classes moyennes blanches, Paterson fait figure de résistant au sein d'une petite communauté urbaine multiraciale et multiculturelle. Le fait qu'il aspire à rejoindre l'auteur de In the American Grain (1925) prend une signification politique particulière en 2016, quand les acquis des années 1960 dans lesquels Jarmusch a grandi semblent bien menacés : le "grain américain », nous dit Jarmusch, dont les films (Mystery Train, USA/Japon, 1989; Dead Man, USA/ Allemange/Japon, 1995 ; Ghost Dog, France/Allemagne/USA/Japon, 1999; The Limits of Control, USA/Japon, 2009) ont toujours donné une large place aux diversités raciales et ethniques, est bel et bien caractérisé par cette diversité. Paterson poursuit en outre l'exploration du couple aux prises avec le quotidien, un thème déjà étudié à travers l'idée de la rétrospection du héros dans Broken Flowers (USA/France, 2005) et de l'immortalité des vampires dans le sublime Only Lovers Left Alive (Allemagne/UK/ France/Grèce/USA/Chypres, 2013). Car le comportement insolite de Laura, qui ne cesse de décorer son domicile en noir et blanc, interpelle quant à la qualité de la relation qu'elle entretient avec Paterson, qui, lui, sort tous les soirs boire un verre en solitaire. Le film propose alors une vision de l'artiste fort différente de celle du vampire reclus de Only Lovers Left Alive: Paterson est ici ancré dans l'expérience du quotidien, un quotidien dans lequel les autres semblent avoir des vies bien plus trépidantes que la sienne (par exemple le couple afro-américain qui rejoue la tragédie de Roméo et Juliet). Mais le statut d'artiste est toujours décrit comme un engagement créatif condamné à la répétition et à l'imperfection, en même temps qu'il profite de la jouissance de l'éternel recommencement.

\section{The Revenant (USA/Hong Kong/Taiwan, Alejandro G. Iñárritu)}

13 The Revenant célèbre le résultat d'une double performance, chacune étant mise au service de l'autre. D'abord la performance d'un comédien surinvesti dans son rôle, comme pouvaient l'être dans les années 70 les élèves de l'Actor's Studio accédant au grand écran. Ensuite la performance formelle d'un cinéaste surinvesti dans la recherche de l'expérience esthétique la plus sensorielle, visant à retrouver là-aussi le geste des cinéastes américains des années 70 - le film d'Inárritu est en quelque sorte le remake de Man in the Wilderness (USA, Richard C. Sarafian, 1971). The Revenant a suscité la suspicion, comme avant lui Birdman (USA, Iñárritu, 2014), en raison d'un argument que l'on est droit de trouver spécieux : celui de la trop grande maîtrise du cinéaste sur son matériau, en l'occurrence en raison de l'aspect jugé m'as-tu-vu à la fois de la mise en scène (avec ses très longs plans-séquences), et du jeu de Di Caprio, à la recherche de la composition la plus oscarisable, d'ailleurs enfin récompensée en 2016. Ce qui aurait manqué à ce western en fin de compte, ce serait sa sincérité, l'âpreté de sa rencontre avec la violente réalité de la nature, comme avaient pu le vivre avant lui William Friedkin, Werner Herzog ou bien sûr Francis Ford Coppola. Si la réussite de l'œuvre ne s'est pas payée au prix d'une épreuve humaine dont les cicatrices seraient perceptibles à l'image, si ses plans ne rendent pas compte des accidents et des insuccès rencontrés lors du tournage, alors tout à l'écran n'y serait que gratuité, artifice, vacuité et vanité. Une telle liquidation du formalisme, d'un tel désamour pour la complète maitrise 
formelle, ou au moins sa revendication, ne fait pourtant que ranimer les débats les plus séculaires sur la dimension ontologique du dispositif, et fait avant tout porter la culpabilité sur l'implication du spectateur devant la puissance d'attraction de la projection cinématographique. Il est à saluer pourtant qu'un réalisateur hollywoodien ait aujourd'hui à cœur d'employer toutes les ressources à sa disposition pour proposer des expériences proprement cinématographiques, c'est-à-dire ici une exploitation immersive du cadre dans le paysage sauvage et une hyperbolisation de la durée, soit un spectacle à vivre pleinement sur grand écran. Car comme les cinéastes du New Hollywood, c'est bien à une synthèse entre grand spectacle et cinéma d'art qu'aspire Iñárritu. The Revenant signale le renouveau du film d'action comme cinéma total, cette fois-ci à travers la mise en cadre plutôt que la mise en chaîne comme dans Mad Max: Fury Road (Australie/USA, George Miller, 2015). Le film affiche alors son désir de s'inscrire à sa façon dans une poétique de la nature, avec ces emprunts à Herzog mais aussi à Andrei Tarkovski, la narration mettant en avant la dimension (méta)physique de l'expérience. Que cette ambition soit parfois "voyante », affichée pour elle-même, que l'ours qui attaque le héros lors d'une éprouvante séquence soit en réalité une créature de synthèse, cela doit d'abord être vu comme la célébration d'une certaine conception du cinéma indifférente aux regards dogmatiques qui cherchent à porter méfiance et diffamation sur le pouvoir envoûtant de l'image.

INDEX

Mots-clés : cinéma, américain, britannique, canadien, néo-zélandais

Thèmes : Film

\section{AUTEURS}

\section{DAVID ROCHE}

Université Toulouse Jean Jaurès

mudrock@neuf.fr

\section{VINCENT SOULADIÉ}

Université Toulouse Jean Jaurès

souladiv@yahoo.fr 